Check for updates

Cite this: RSC Adv., 2019, 9, 1551

Received 6th December 2018

Accepted 6th January 2019

DOI: $10.1039 / c 8 r a 10045 d$

rsc.li/rsc-advances

\section{Multi-scale instrumental analyses of plasticized polyhydroxyalkanoates (PHA) blended with polycaprolactone (PCL) and the effects of crosslinkers and graft copolymers $\dagger$}

\author{
Masakazu Nishida, (D) *a Tomoko Tanaka, ${ }^{a}$ Yoshio Hayakawa, (D) a Takashi Ogura, ${ }^{\mathrm{b}}$ \\ Yoshiaki Ito ${ }^{\mathrm{b}}$ and Masahiro Nishida (D) ${ }^{\mathrm{b}}$
}

\begin{abstract}
Details of the mechanism underlying the tensile properties of plasticized polyhydroxyalkanoates (PHA) including poly(butylene succinate) (PBS) were investigated by blending with poly( $\varepsilon$-caprolactone) (PCL) as well as the addition of compatibilizers. Multi-scale instrumental analyses employed micro-focus X-ray CT to provide micro-scale morphology information on the order of ten microns while solid-state NMR spectral and relaxation time analyses contributed knowledge of the environment and molecular mobility of each constituent at the molecular to nano-scale. The blend of plasticized PHA with $50 \%$ PCL adopted a sea-island morphology to improve elongation at break in a quasi-static tensile test, which was dominated by the tensile properties of the added PCL. However, impact tensile properties were less improved by PCL addition, because its molecular mobility was suppressed by blending. Meanwhile, peroxy crosslinkers changed the sea-island morphology to homogenous in X-ray CT observations. Although the homogenous morphology sharply lowered the elongation at break in a quasi-static tensile test, the homogenous morphology improved impact tensile properties. Furthermore, graft polymers having acrylonitrile-styrene side-chains did not change the sea-island morphology but increased the molecular mobility of PBS in the plasticized PHA. This weak interaction between the plasticized PHA and PCL improved tensile properties in both quasi-static and impact tensile tests.
\end{abstract}

\section{Introduction}

As part of the solution to global climate problems for a sustainable society, it is desirable to replace petroleum-based polymers with biodegradable equivalents. Polyhydroxyalkanoates (PHA), which are made by a microbial process, have the advantage of consuming non-edible materials as the feedstocks. ${ }^{1}$ PHA is a family of biopolyesters commonly accumulated by many bacteria, consisting of the following polyesters: poly-3-hydroxybutylate (PHB), poly-3-hydroxyvalerate (PHV), as well as their isomeric polymers and copolymers. The control of chemical structure of the PHA homopolymers and copolymers in both microbial and chemical production is still under development. ${ }^{2}$ Applications of PHA have extended to various industrial fields with developments in characterization and production. In particular, PHA is useful in medical sectors,

${ }^{a}$ National Institute of Advanced Industrial Science and Technology (AIST), 2266-98 Shimoshidami, Moriyama-ku, Nagoya, 463-8560 Japan.E-mail: m-nishida@aist.go. jp; Fax: +81 52736 7406; Tel: +81 527367493

${ }^{b}$ Nagoya Institute of Technology, Gokiso-cho, Showa-ku, Nagoya, Aichi, 466-8555, Japan

$\dagger$ Electronic supplementary information (ESI) available. See DOI: $10.1039 / \mathrm{c} 8 \mathrm{ra} 10045 \mathrm{~d}$ such as tissue engineering, bio-implants, drug delivery, and surgical materials, as well as in nanotechnology. ${ }^{3}$ Other industrial applications include packaging, moulded goods, paper coatings, non-woven fabrics, adhesives, films, and performance additives. ${ }^{4}$ Considering these applications, the chemomechanical properties of PHA, which are influenced by polymer composition and microstructure, have been investigated. ${ }^{5}$

Chemical modification approaches can alter the characteristics of PHA, such as its mechanical properties, surface structure, amphiphilic character, and rate of degradation. ${ }^{6}$ Hydroxylation, carboxylation, epoxidation, and chlorination in particular have been considered for use in biomedical applications. ${ }^{7}$ Blending of PHA with other polymers is the most important method for controlling its thermal and crystallization behaviour and morphology which are closely related to mechanical properties and biodegradability. ${ }^{8}$ The morphology of poly(3-hydroxybutyrate-co-3-hydroxyvalerate) (PHBV)/ polylactide (PLA) blends was studied to assess the correlation to thermal, rheological and barrier properties. ${ }^{9}$ Depending on the application, bio-based PLA/PHB blend can be manufactured in various shapes. Plasticized PLA/PHB blends with an oligomer of lactic acid, which was extruded for the film formation process, showed a decrease in the glass transition temperature 
while maintaining the mechanical properties. ${ }^{10}$ The plasticized PLA/PHB blend could be also produced as flexible fibres by the electrospinning method and the blend ratio affected the biodegradability of the electrospun fibres in composting conditions due to their crystallinities. ${ }^{11}$ In order to improve melt processability and thermomechanical properties, PHA was blended with poly(butylene adipate-co-terephthalate) (PBAT) by melt extrusion to demonstrate that an acid wash dramatically improved processability. ${ }^{12}$ Binary blends for PHB and poly( $\varepsilon-$ caprolactone) (PCL) blend that were manufactured by a twin screw co-rotating extruder and were subsequently injection moulded showed impact that depended on the PCL content. ${ }^{13}$

Since PHA is brittle and hydrophilic, it has disadvantages for processability and a small amount of compatibilizer has sometimes been used in blending PHA; the morphology and mechanical properties of the PHA-based blend were changed by compatibilizers, including crosslinkers and graft copolymers. The reactive polymer blending technique changed the physicochemical interactions between the constituent polymers because of in situ formation of the compatibilizing agents, such as a branching/crosslinking copolymer and a graft copolymer. ${ }^{14}$ An epoxy compatibilizer reacted with the $\mathrm{OH}$ group of poly(3hydroxybutyrate-co-3-hydroxyhexanoate) (PHBH) and the $\mathrm{OH} /$ $\mathrm{COOH}$ groups of PLA to improve the elongation at break and impact strength of the PHBH/PLA blend..$^{15}$ Dicumyl peroxide, which acted as a free-radical grafting initiator, compatibilized PHBV/PBS and PHA/PBS blends to ameliorate tensile strength, impact toughness, and elongation at break of injectionmoulded blends. ${ }^{16}$ Grafting maleic anhydride improved the miscibility of a PHBV/PBS blend with incorporation of sepiolite because of a synergistic effect induced by both compatibilizer and filler. ${ }^{17}$ Starch-based materials can be used as compatibilizer for melt-blending with PHA and PBAT, inhibiting the secondary crystallization of the PHA component in the cast films. Such approaches show promise for flexible packing materials. ${ }^{18}$

As shown above, the blending of PHA as well as the compatibilizing of PHA-based blends obviously improved the material characteristics of PHA; however, in order to know the details of the mechanism of their functional expression, an analytical method that covers molecular- to nano-scale length scales is necessary. Solid-state NMR is a useful analytical method in particular for both synthetic and natural polymers over these small-scale orders; it has provided significant information about molecular structures and dynamics for amorphous and crystalline phases of $\mathrm{PHB}^{19}$ as well as PHBbased copolymers and blends. ${ }^{20,21}$ Despite the correlation of microscopic properties to not only the morphology, but also to the mechanical properties of PHA and related materials, solidstate NMR has not yet been applied to analyses of structures and dynamics from the molecular- to nano-scale orders. Using the multi-scale instrumental analyses that range from molecular- to nano-scale (solid-state NMR) to micro-scale (morphology by SEM observation), we have evaluated the material characteristics of biomass polymers, for example, changes of biomass constituents of soft wood ${ }^{22}$ and other plant materials. ${ }^{23}$
The present study aims to extend multi-scale instrumental analyses to the multicomponent PHA system to reveal changes at the molecular- to micro-scales that contribute to their mechanical properties. Our preceding paper has revealed the influence of molecular mobility on the compatibility of PHA and PBS for plasticized PHA (commercially available PHA4422P), using solid-state NMR spectral and relaxation time analyses not only at ambient and variable temperatures. ${ }^{24}$ In a quasi-static tensile test of this plasticized PHA blending with PCL, the addition of 50\% PCL dramatically increased the elongation at break and the specimens became pours shape. ${ }^{25}$ In a dynamic tensile test using a split Hopkinson bar (SHPB) method, a blend consisting of 50\% plasticized PHA and 50\% PCL gave small elongation at break, which increased with the addition of compatibilizers. ${ }^{26}$ Here, we focus on plasticized PHA (PHA4422P) blended with various amounts of PCL and with various compatibilizers (crosslinkers and graft polymers). By combining multi-scale instrumental analyses including solid-state NMR spectral and relaxation time analyses and micro-focus X-ray computed tomography (CT) with newly collected systematic tensile test data, the details of the mechanism behind their mechanical properties have been elucidated.

\section{Experimental}

\section{Materials}

The plasticized PHA pellet, PHA4422P, was purchased from G5 Manufacturing, Singapore. The PHA4422P pellet consisted of $65 \%$ PHA, 30\% PBS, and 5\% crosslinking reagent. The PCL pellet, Capa 6800, was purchased from Perstorp Japan Co. Ltd., Tokyo, Japan. The crosslinking reagents, PERHEXA 25B (liquid, over $90 \%$ purity) and PERHEXA 25B-40 (mixed with silica as a white solid, $40 \%$ purity), were purchased from NOF CORPORATION, Tokyo, Japan. The graft polymers, Modiper A4400 and Modiper CL430-G, were purchased from NOF CORPORATION, Tokyo, Japan. Modiper A4400 was an ethylene-glycidyl methacrylate copolymer with acrylonitrile-styrene copolymer. Modiper CL430L was polycarbonate with a functional groupmodified acrylonitrile-styrene copolymer. All materials were used without further purification. Raw pellets and compatibilizers ( $1 \%$ crosslinkers and $1 \%$ graft polymers) were melt-mixed at $175{ }^{\circ} \mathrm{C}$ for $20 \mathrm{~min}$ at a rotor speed of $50 \mathrm{rpm}$ with a conventional melt-mixer. The mixed sample was moulded into plates of $5 \mathrm{~mm}$ thicknesses at $175^{\circ} \mathrm{C}$ and $30 \mathrm{MPa}$ for $30 \mathrm{~min}$ with a hot press.

\section{Static tensile test}

Static tensile test specimens with a gauge area of $5 \mathrm{~mm} \times 2 \mathrm{~mm}$ and a gauge length of $10 \mathrm{~mm}$ were made from the plates using machine processing. Static tensile tests were carried out at a crosshead speed of $0.2 \mathrm{~mm} \mathrm{~min}^{-1}$ using a universal testing machine. Nominal strain was obtained by images of the gauge length, taken by a digital camera in the elastic region and by the displacement of the crosshead in the plastic region. Nominal 
stress was calculated using the output of load cell at the crosshead.

\section{Dynamic tensile test}

Dynamic tensile test specimens with a gauge area of $2 \mathrm{~mm} \times$ $5 \mathrm{~mm}$ and a gauge length of $4 \mathrm{~mm}$ were made from the plates using machine processing. At high strain rates, the dynamic properties of the specimens were examined by the tensile split Hopkinson bar test. ${ }^{27}$ The diameters and lengths of input and output bars were $12 \mathrm{~mm}$ and $2000 \mathrm{~mm}$ respectively. Strain gauges were applied to both sides of the input and output bars at distances of $1750 \mathrm{~mm}$ and $350 \mathrm{~mm}$ from the specimen, respectively.

\section{Micro-focus X-ray CT observations}

Micro-focus X-ray images were recorded with a FLEX-M345CT (Beamsense Co. Ltd., Osaka, Japan) instrument at an acceleration voltage of $40 \mathrm{kV}$ and a tube current of $100 \mu \mathrm{A}$. The sample was rotated over $180^{\circ}$ at $0.5^{\circ}$ steps and two transmission images (the pixel size is $2.12 \mu \mathrm{m} \times 2.12 \mu \mathrm{m}$ to $3.07 \mu \mathrm{m} \times 3.07 \mu \mathrm{m}$ ) were acquired using a charge-coupled device (CCD) with the exposure time of $1500 \mathrm{~ms}$. The CT images were obtained by slicing the 3D image reconstructed from the transmission images.

\section{Solid-state NMR spectrum measurements}

Magic angle spinning (MAS) nuclear magnetic resonance (NMR) spectra were measured on a Varian 400 NMR system spectrometer (Palo Alto, CA) with a Varian $4 \mathrm{~mm}$ double-resonance T3 solid probe. The samples were placed in a $4 \mathrm{~mm} \mathrm{ZrO}_{2}$ rotor spun at $15 \mathrm{kHz}$ over a temperature range of $20-22{ }^{\circ} \mathrm{C} .{ }^{1} \mathrm{H}$ MAS NMR spectra were measured at $399.86 \mathrm{MHz}$ for the ${ }^{1} \mathrm{H}$ nuclei and were collected with a $40 \mathrm{~ms}$ acquisition period over a $30.5 \mathrm{kHz}$ spectral width in 16 transients, and a $3 \mathrm{~s}$ recycle delay. ${ }^{13} \mathrm{C}$ MAS NMR spectra were collected with a $2.6 \mu \mathrm{s} \pi / 2$ pulse at $100.56 \mathrm{MHz}$ for the ${ }^{13} \mathrm{C}$ nuclei and a $40 \mathrm{~ms}$ acquisition period over a $30.7 \mathrm{kHz}$ spectral width. Proton decoupling was performed with an $86 \mathrm{kHz}^{1} \mathrm{H}$ decoupling radio frequency with a small phase incremental alteration (SPINAL) decoupling pulse sequence. Cross-polarization/magic angle spinning (CP-MAS) NMR spectra were measured with a $5.0 \mathrm{~s}$ recycle and 1024 transients delay, using a ramped-amplitude pulse sequence with a $2 \mathrm{~ms}$ contact time and a $2.5 \mu \mathrm{s} \pi / 2$ pulse for the ${ }^{1} \mathrm{H}$ nuclei. The amplitude of the ${ }^{1} \mathrm{H}$ pulse was ramped up linearly from $90.5 \%$ of its final value during the cross polarization contact time. Pulse saturation transfer/magic angle spinning (PST-MAS) NMR was measured used the single $\pi / 2$ pulse for the ${ }^{13} \mathrm{C}$ nuclei with a $5 \mathrm{~s}$ recycle delay in 2048 transients after saturation of ${ }^{1} \mathrm{H}$ nuclei with 13 consecutive $2.5 \mu$ s pulses and a $27.5 \mu$ s delay.

\section{Nuclei magnetic relaxation analyses}

The ${ }^{1} \mathrm{H}$ spin-lattice relaxation time in the laboratory frame $\left(T_{1} \mathrm{H}\right)$ was indirectly measured via detection of ${ }^{13} \mathrm{C}$ resonance enhanced by cross-polarization, applied after a $\pi$ pulse to ${ }^{1} \mathrm{H}$ nuclei with the inversion recovery method. The ${ }^{13} \mathrm{C}$ spin-lattice relaxation time in the laboratory frame $\left(T_{1} \mathrm{C}\right)$ was measured with the conventional Torchia's pulse sequence. ${ }^{28}$ The $T_{1}$ analyses were performed with the same solid-state probe with the same contact time and acquisition period used for the ${ }^{13} \mathrm{C}$ CP-MAS NMR spectrum. The ${ }^{1} \mathrm{H}$ spin-lattice relaxation in the rotation frame $\left(T_{1 \rho} \mathrm{H}\right.$ relaxation) was examined by the observation of signal decay curve with variable contact time over a range of 100-12 $000 \mu \mathrm{s}$ in CP-MAS NMR sequence.

\section{Results and discussion}

Effects of PCL ratio and compatibilizers on tensile properties of plasticized PHA/PCL blends

As shown in our previous paper, ${ }^{25}$ the tensile strength and elongation at break in a quasi-static tensile test were significantly changed by addition of PCL to the plasticized PHA, PHA4422 (PHA/PBS copolymer), depending on the PCL ratio. In particular, over $50 \%$ PCL addition increased both the strength and elongation in the quasi-static test. In contrast, the value of the PCL ratio scarcely influenced the impact tensile test of the plasticized PHA/PCL blend. The different effects of the PCL ratio and compatibilizers between the quasi-static and impact tensile tests can be observed in Fig. 1. Comparing the quasi-static (Fig. 1A) and impact (Fig. 1B) tests, the 100\% PCL specimen has a smaller elongation at break in the impact test than that in the quasi-static test. The plasticized PHA/PCL blend showed less difference with the PCL content in the impact test than in the quasi-static test, owing to the above tensile properties of PCL.

The tensile strength and elongation at break of the $50 \%$ plasticized PHA $/ 50 \%$ PCL blend in the impact test noticeably increased with only $1 \%$ compatibilizers, such as the crosslinkers (PERHEXA 25B, PERHEXA 25B-40) and graft polymers (Modiper A4400, Modiper CL430L), as previously communicated ${ }^{26}$ In the preliminary experiments, the addition amount of the compatibilizer was not greatly different between $1 \%$ and $5 \%$. Thus, the addition amount of the compatibilizer was examined for $1 \%$ in this study. The additives produced different effects in the quasi-static (Fig. 1C) and impact (Fig. 1D) tests, as well as with changes of the PCL ratio. In the quasi-static test, the crosslinkers significantly decreased elongation at break while they increased tensile strength. The graft polymers in the quasistatic test also increased tensile strength, but they only slightly decreased elongation at break compared with the original blend.

\section{Morphological observations of plasticized PHA/PLA blends with micro-focus X-ray CT}

In order to examine changes of 50\% plasticized PHA (PHA/PCL copolymer) $/ 50 \%$ PCL blend due to the compatibilizers in terms of micro-scale order, morphological changes of specimens after quasi-static and impact tensile tests were observed using softfocus X-ray CT for specimens having fractures of different shapes. The morphological difference of $50 \%$ plasticized PHA/ $50 \%$ PCL blend after the quasi-static and impact tests is shown in Fig. 2. One can see that a sea-island structure having relatively large island domains $(<1000 \mu \mathrm{m})$ was observed in both 
A

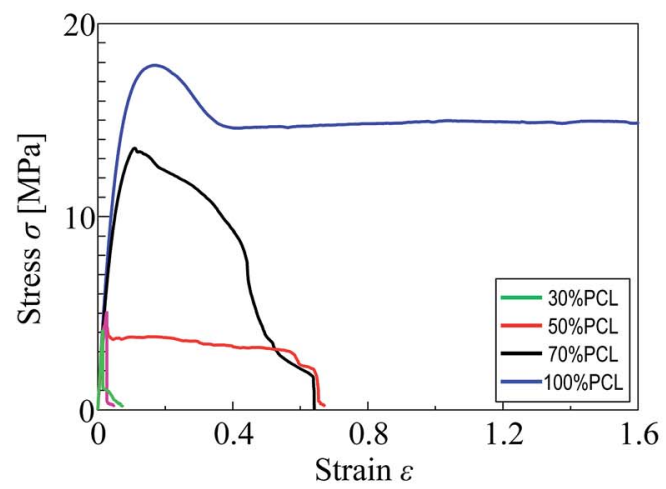

B

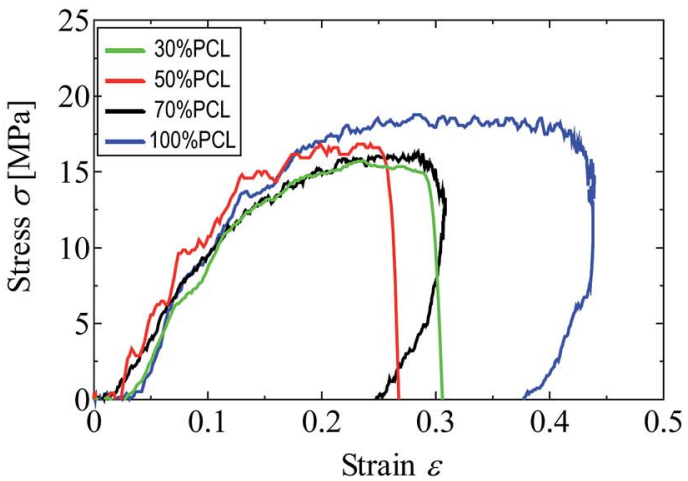

C

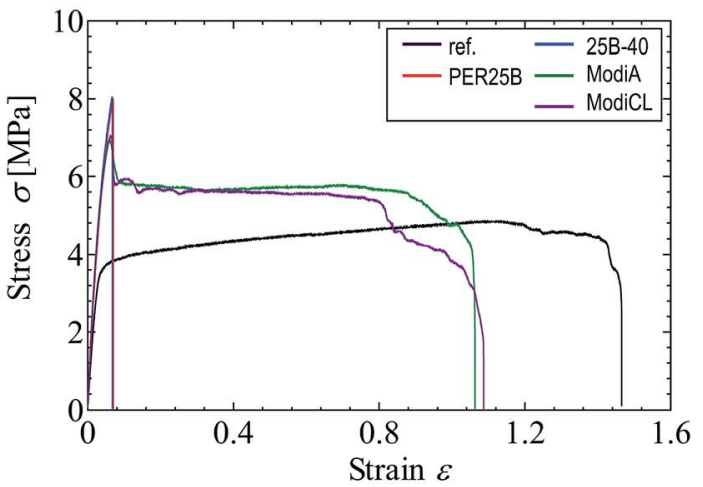

D

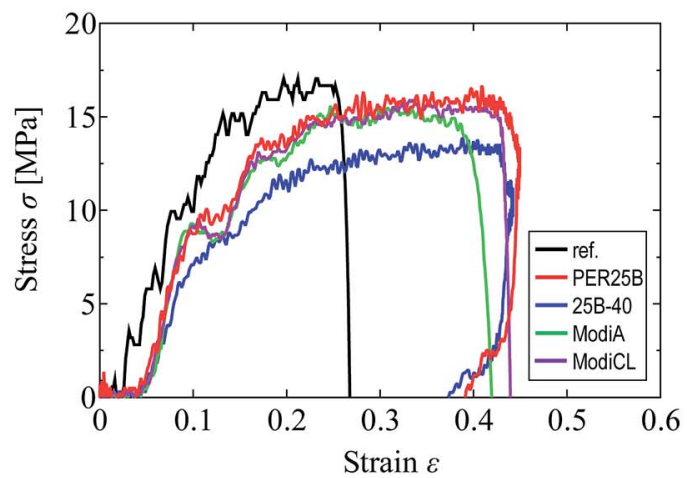

Fig. 1 Quasi-static tensile test and impact tensile test of plasticized PHA/PCL blends. (A) Quasi-static (plasticized PHA/PCL blend). (B) Impact (plasticized PHA/PCL blend). (C) Quasi-static (50\% PCL with compatibilizers). (D) Impact (50\% PCL with compatibilizers).

specimens. In the specimen after quasi-static test (Fig. 2A), the island portion was broken to several sections while the sea portion was stretched with minute voids. This morphology after the quasi-static test corresponded to the porous structure previously observed in the visible and low magnification X-ray CT images. ${ }^{25}$ In contrast, after the impact test (Fig. 2B), both
A

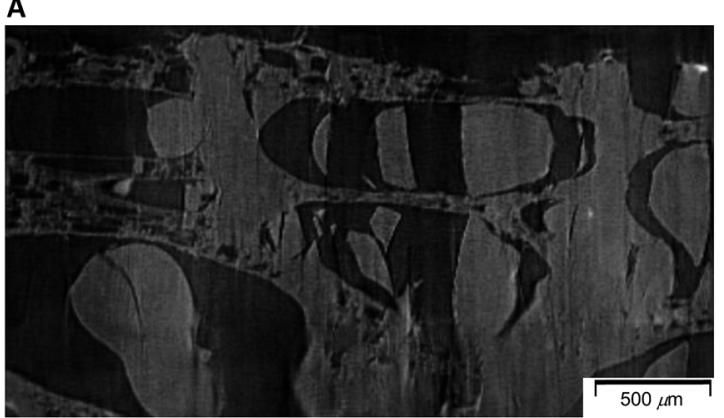

B

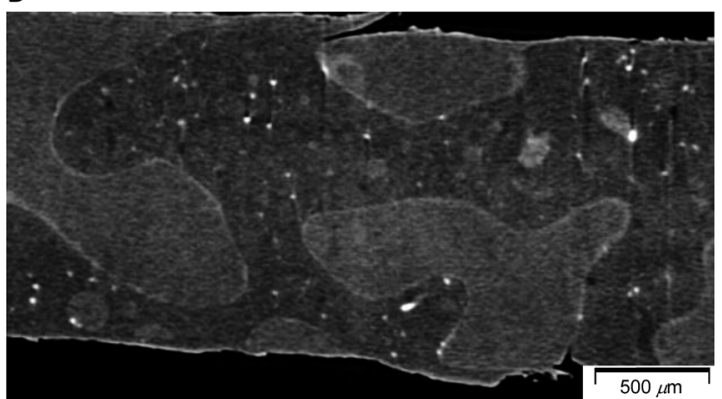

C

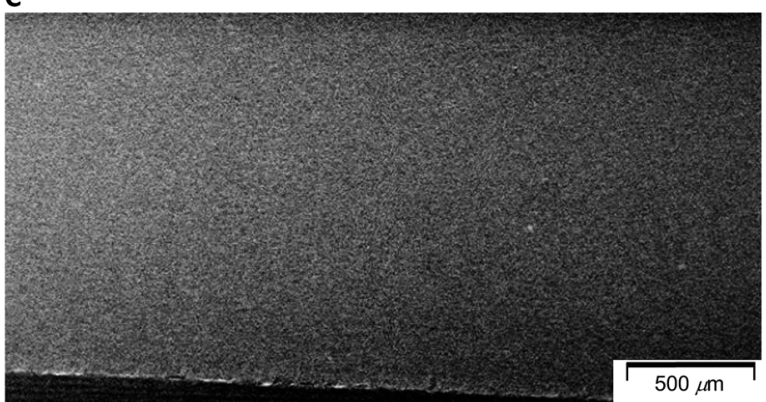

D

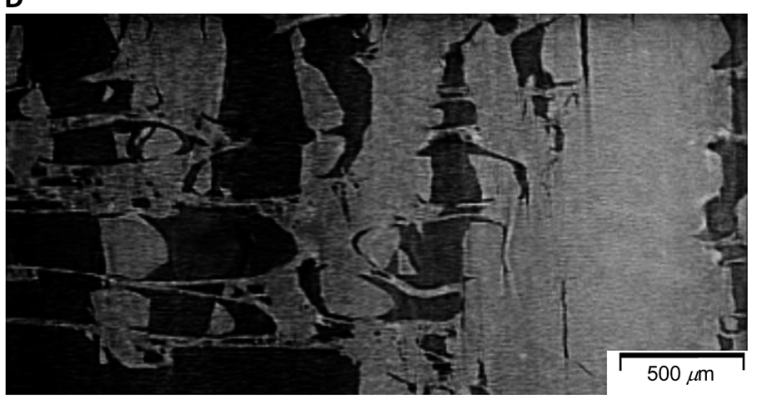

Fig. 2 Micro-focus X-ray CT images of 50\% plasticized PHA/50\% PCL blends. (A) Without compatibilizers after quasi-static test. (B) Without compatibilizers after impact test. (C) With PERHEXA25B after quasi-static test. (D) With Modiper A4400 after quasi-static test. 
island and sea shapes were not deformed and showed almost no stretching. Since the $100 \%$ PCL specimen has a large elongation at break in the quasi-static test while it had a small elongation in the impact test, the sea portion was assigned as PCL and the island portion was assigned as PHA.

Since the effect of compatibilizers appeared in the quasistatic tensile test more clearly, morphological changes of the $50 \%$ plasticized PHA $/ 50 \%$ PCL blend due to the compatibilizers were examined for the specimens after the quasi-static test using micro-focus X-ray CT. The crosslinker (1\% PERHEXA 25B) changed the sea-island structure of plasticized PHA/PCL blend into a homogenous morphology over tens of micron (Fig. 2C). In this case, neither visible cracks nor deformations were observed, being accountable for the very small elongation at break in the quasi-static test. Meanwhile, the graft polymer (1\% Modiper A4400) maintained the sea-island structure of the plasticized PHA/PCL blend; however, both the island and sea regions were broken and elongation of sea portion was also observed at the same time (Fig. 2D). Therefore, the graft polymer enhanced the interaction between plasticized PHA and PCL resulting in the fracture of the sea portion consisting of PCL caused by low elongation of PHA.

\section{${ }^{1}$ H MAS NMR spectra of plasticized PHA/PLA blends}

Since ${ }^{1} \mathrm{H}$ nuclei have large dipolar-dipolar interactions with each other, solid-state ${ }^{1} \mathrm{H}$ NMR spectra have wide lines even when using magic-angle spinning (MAS), which can offset the anisotropy of dipoles. The line width of ${ }^{1} \mathrm{H}$ MAS NMR spectra is affected by the spin-spin relaxation ( $T_{2}$ relaxation) which is suppressed by molecular motions. For example, water molecules had a narrower line width because of a longer $T_{2}$ (greater molecular mobility) while biomass constituents in soft wood showed a wider line shape because of shorter $T_{2}$ (less molecular mobility). ${ }^{29}$ Here, the molecular mobility involved in $T_{2}$ relaxation could be monitored by ${ }^{1} \mathrm{H}$ MAS NMR spectra of plasticized PHA/PCL blends.

First, changes of ${ }^{1} \mathrm{H}$ MAS NMR spectra of plasticized PHA/ PCL blends with PCL ratio are shown in Fig. 3A. Although both pre-plasticized PHA and PBS copolymer had broad lowintensity signals as shown in Fig. S1 (ESI $\dagger$ ), the plasticized PHA (PHA4422P) showed relatively sharp signals not only in the PHA region but also in the PBS region [Fig. 3A(1)]. Therefore, the plasticization of PHA with PBS enhanced molecular motions of both PHA and PBS because of the suppression of $T_{2}$ relaxation. Like the plasticized PHA, flexible PCL showed sharp highintensity signals [Fig. 3A(5)]. All plasticized PHA blends including $30 \%, 50 \%$, and 70\% PCL [Fig. 3A(2)-(4)] also showed similar line shapes and intensities to the plasticized PHA and flexible PCL. Thus, molecular mobility concerned with $T_{2}$ relaxation was little changed by blending with flexible PCL because the copolymerization with PBS has already amplified the molecular mobility of the constituent polymers.

Next, changes of ${ }^{1} \mathrm{H}$ MAS NMR spectra of the $50 \%$ plasticized PHA/50\% PCL blend with the addition of compatibilizers are shown in Fig. 3B. Based on the signal shapes and intensities of $50 \%$ plasticized $\mathrm{PHA} / 50 \%$ PCL blend without additives [Fig. 3B(a)], the compatibilizers scarcely changed the ${ }^{1} \mathrm{H}$ signal shape except that the addition of PERHEXA25B-40 slightly changed the amount of overlapping ${ }^{1} \mathrm{H}$ signals [Fig. $3 \mathrm{~B}(\mathrm{c})$ ]. Therefore, changing the PCL ratio and the addition of both crosslinkers and graft polymers scarcely changed the molecular mobility involved in $T_{2}$ relaxation.

\section{${ }^{13}$ C MAS NMR spectra of plasticized PHA/PCL blends}

We have already evaluated the crystallinity and mobility of the plasticized PHA copolymers and their constituent polymers
A
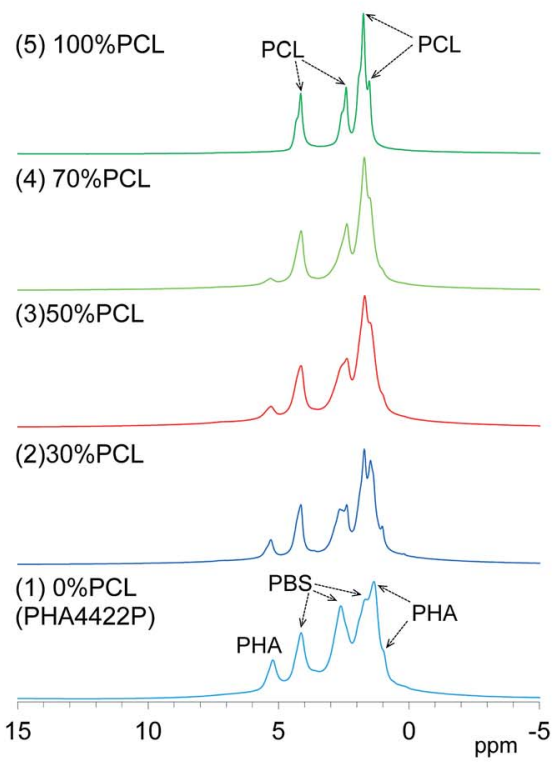

B

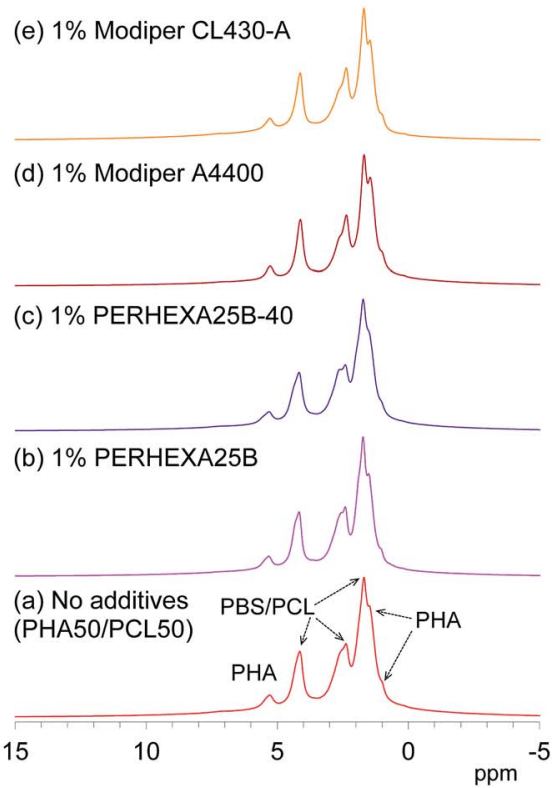

Fig. $3{ }^{1} \mathrm{H}$ MAS NMR spectral changes of plasticized PHA/PCL blends. (A) PCL ratio. (B) Compatibilizers. 
using a combination of ${ }^{13} \mathrm{C}$ CP-MAS NMR and ${ }^{13} \mathrm{C}$ PST-MAS NMR methods. ${ }^{24}$ The former CP-MAS method enhances signals of rigid portions utilizing magnetization transfer from ${ }^{1} \mathrm{H}$ nuclei to ${ }^{13} \mathrm{C}$ nuclei. The latter PST-MAS method enhances signals of flexible portions near hydrogen atoms using the Nuclear Overhauser Effect (NOE). ${ }^{23}$ Here, molecular mobility changes due to the blending ratio and compatibilizers were examined by comparison of CP-MAS and PST-MAS signal intensities for each substituent.

Fig. 4A shows changes of ${ }^{13} \mathrm{C}$ CP-MAS NMR spectra of plasticized PHA/PCL blends with varying PCL content and Fig. 4B shows their ${ }^{13} \mathrm{C}$ PST-MAS NMR spectra. As described in our previous report, the plasticized PHA (PHA4422P) gave sharp signals for the PHA and PBS constituents, which were readily assigned in the ${ }^{13} \mathrm{C}$ CP-MAS NMR spectrum [Fig. 4A(1)]. Since PHA was more rigid than $\mathrm{PBS}$, the $\mathrm{C}=\mathrm{O}$ and $\mathrm{CH}$ signals of the PHA moiety in the ${ }^{13} \mathrm{C}$ PST-MAS NMR spectrum [Fig. $4 \mathrm{~B}(1)$ ] showed a lower ratio of plasticized PHA to PCL than did the ${ }^{13} \mathrm{C}$ CP-MAS NMR spectrum. However, the $\mathrm{CH}_{3}$ signal of the PHA moiety in the ${ }^{13} \mathrm{C}$ PST-MAS NMR spectrum had a high intensity in a similar manner to the PBS moiety because the $\mathrm{CH}_{3}$ group has a high molecular mobility even in rigid polymer like PHA. For flexible polymer such as PCL, the ${ }^{13} \mathrm{C}$ CP-MAS NMR spectrum [Fig. 4A(5)] showed one $\mathrm{C}=\mathrm{O}$ and four $\mathrm{CH}_{2}$ signals, of which the highest field signal was assigned as an overlapped signal of two $\mathrm{CH}_{2}$ groups. In the ${ }^{13} \mathrm{C}$ PST-MAS NMR spectrum [Fig. $4 \mathrm{~B}(5)$ ], the overlapped signal could be separated and the intensities of signals increased with the result that PCL showed six sharp signals.

In the ${ }^{13} \mathrm{C}$ CP-MAS NMR spectra of the plasticized PHA/PCL blends [Fig. 4A(2)-(4)], the PCL signals except that at $34 \mathrm{ppm}$ $\left(\alpha-\mathrm{CH}_{2}\right)$ overlapped with the signals derived from PBS in the plasticized PHA. In the ${ }^{13} \mathrm{C}$ PST-MAS NMR spectra of the plasticized PHA/PCL blends [Fig. 4B(2)-(4)], the PCL signals except for $\alpha-\mathrm{CH}_{2}$ still overlapped with the PBS signals. Therefore, the molecular mobility and dynamics of the plasticized PHA/PCL blend is best discussed using the PHA signals and the $\alpha-\mathrm{CH}_{2}$ signal of PCL. At the same time, the molecular mobility and dynamics of PBS in the plasticized PHA/PCL blend can be discussed by comparison of the $\alpha-\mathrm{CH}_{2}$ of PCL and other overlapped PCL/PBS signals. Although the PHA signals in both the ${ }^{13} \mathrm{C}$ CP- and PST-MAS NMR spectra decreased with increasing the PCL content of the plasticized PHA/PCL, the ratio of PSTMAS signal to CP-MAS signal remained almost constant. In contrast, the ${ }^{13} \mathrm{C}$ PST-MAS NMR signal of the $\alpha-\mathrm{CH}_{2}$ of homopolymer (5) had a higher intensity than the ${ }^{13} \mathrm{C}$ CP-MAS NMR signal. This ratio of PST-MAS signal to CP-MAS signal was reduced by blending with the plasticized PHA. Since PST-MAS emphasizes signals of flexible portions, this result indicates that the blending with the plasticized PHA suppressed the molecular mobility of PCL.

The effects of the compatibilizers on molecular mobility were also evaluated by the combination of ${ }^{13} \mathrm{C}$ CP-MAS NMR and ${ }^{13} \mathrm{C}$ PST-MAS NMR methods. Fig. 5A shows changes of the ${ }^{13} \mathrm{C}$ CP-MAS NMR spectra of the $50 \%$ plasticized PHA/50\% PCL blend with the compatibilizers and Fig. 5B their ${ }^{13} \mathrm{C}$ PST-MAS NMR spectra. The addition of graft polymers (Modiper A4400, Modiper CL430-G) decreased the $\mathrm{C}=\mathrm{O}$ signal intensity of PBS/ PCL in the ${ }^{13} \mathrm{C}$ CP-MAS NMR spectra [Fig. 5A(d) and (e)], compared with the ${ }^{13} \mathrm{C}$ PST-MAS NMR spectra [Fig. 5B(d) and (e)], even though the crosslinkers (PERHEXA 25B, PERHEXA 25B-40) exhibited smaller differences between CP-MAS [Fig. 5A(b) and (c)] and PST-MAS [Fig. 5B(b) and (c)]. The combination of the two ${ }^{13} \mathrm{C}$ MAS NMR methods showed that the compatibilizers scarcely changed the molecular mobility involved in NOE except that the graft polymers slightly
A

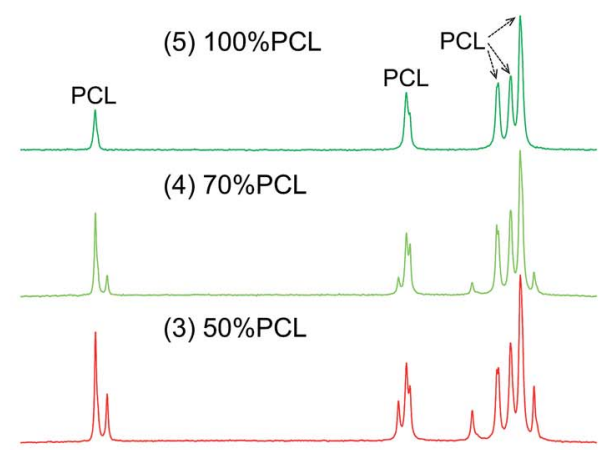

(2) $30 \% \mathrm{PCL}$

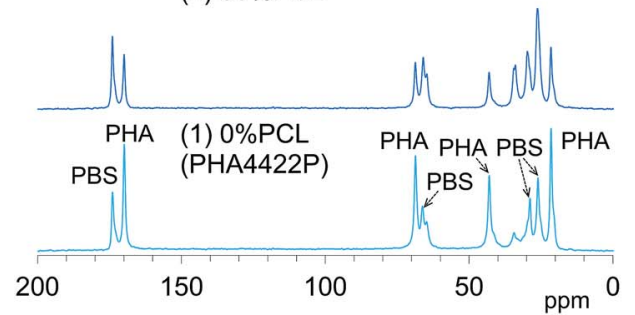

B

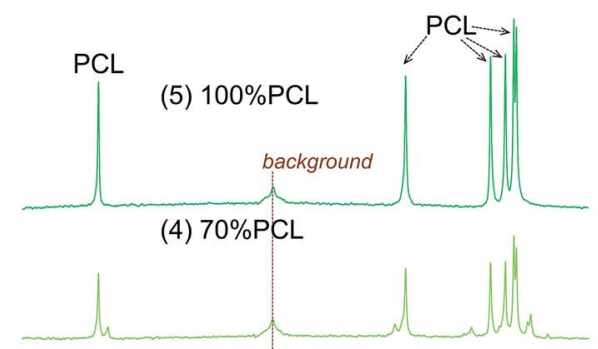

(3) $50 \% \mathrm{PCL}$

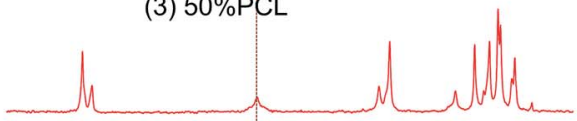

(2) $30 \% \mathrm{PCL}$

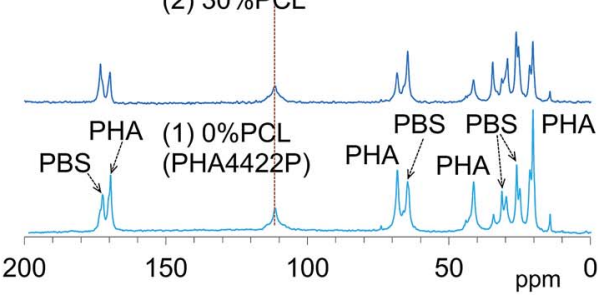

Fig. $4{ }^{13} \mathrm{C}$ MAS NMR spectral changes of plasticized PHA/PCL blends with PCL ratio. (A) ${ }^{13} \mathrm{C}$ CP-MAS NMR. (B) ${ }^{13} \mathrm{C}$ PST-MAS NMR. 
A

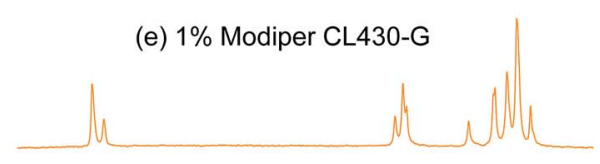

(d) 1\% Modiper A4400

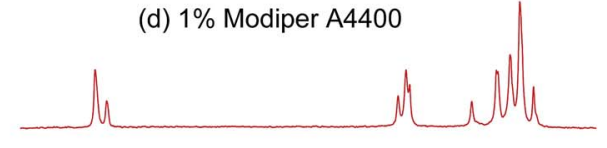

(c) $1 \%$ PERHEXA25B-40

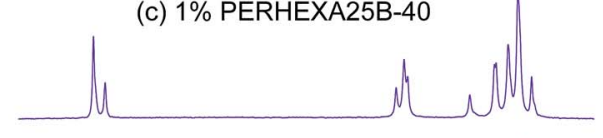

(b) $1 \%$ PERHEXA25B

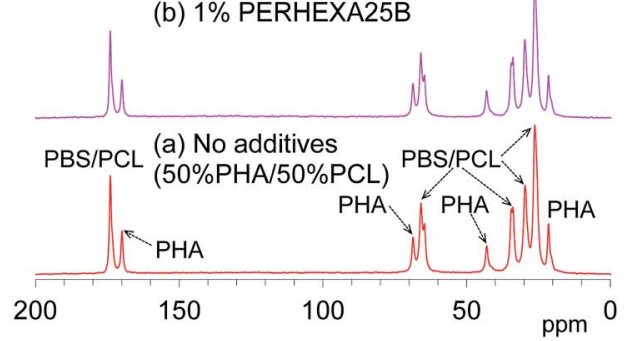

B

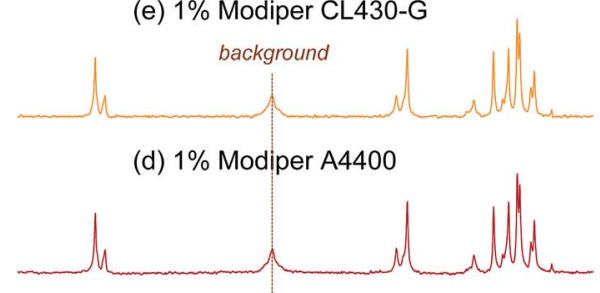

(c) 1\% PERHEXA25B-40

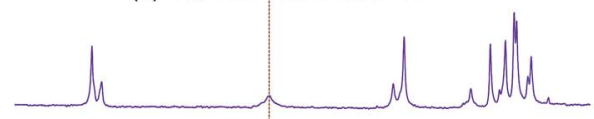

(b) $1 \%$ PERHEXA25B

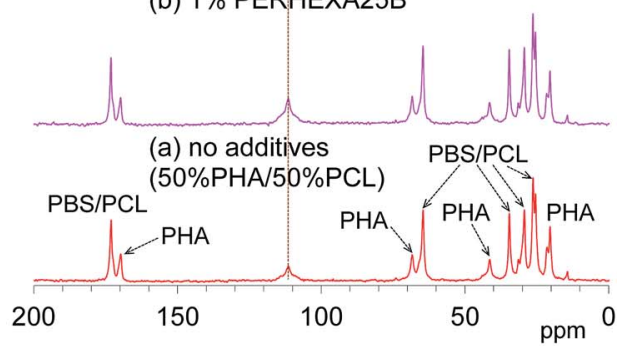

Fig. $5{ }^{13} \mathrm{C}$ MAS NMR spectral changes of $50 \%$ plasticized PHA/50\% PCL blend with compatibilizers. (A) ${ }^{13} \mathrm{C}$ CP-MAS NMR. (B) ${ }^{13} \mathrm{C}$ PST-MAS NMR.

enhanced the molecular mobility of the $\mathrm{C}=\mathrm{O}$ groups of PBS/ PCL.

\section{Spin-lattice relaxation in the laboratory frame of plasticized PHA/PCL blends}

As shown in our preceding study, with increasing the measurement temperature, the $T_{1} \mathrm{H}$ values of pre-plasticized PHA increased while the $T_{1} \mathrm{H}$ values of PBS homopolymer decreased. In the plasticized PHA (PHA/PBS copolymer), however, the $T_{1} \mathrm{H}$ values of the PHA moiety were shortened by the copolymerization with PBS at a higher temperature, because the $T_{1} \mathrm{H}$ relaxation of PHA moiety was enhanced via $\mathrm{PBS}$, which had shorter $T_{1} \mathrm{H}$ value than the PHA moiety. ${ }^{24}$ On the other hand, the $T_{1} \mathrm{H}$ value of PCL homopolymer decreased with increasing temperature as did that of the PBS homopolymer. As shown in Fig. S2 (ESI $\dagger$ ), the PCL (5 : 100\% PCL) showed shorter $T_{1} \mathrm{H}$ values than the constituent polymers in the plasticized PHA (1: $0 \%$ PCL).

First we examined the effects of the blending with PCL on the $T_{1} \mathrm{H}$ value change for each substituent in the plasticized PHA/ PCL (Fig. 6A). Even though PCL had shorter $T_{1} \mathrm{H}$ values than the plasticized PHA, the $T_{1} \mathrm{H}$ values of the PHA moiety in the plasticized PHA/PCL blends were increased by the addition of PCL. The $T_{1} \mathrm{H}$ values of PHA moiety attained a maximum value at $50 \%$ PCL addition (3). According to the results of the microfocus X-ray CT, the increase in the $T_{1} \mathrm{H}$ values of the PHA moiety were caused by the change to the sea-island morphology. That is, the $T_{1} \mathrm{H}$ relaxation of PHA, accelerated by the addition of PBS, was suppressed by the sea-island morphology. A similar situation applies to the PCL moiety $\left(\alpha-\mathrm{CH}_{2}\right)$ in the plasticized PHA/ PCL blends, where $T_{1} \mathrm{H}$ values increased with an increasing fraction of PHA (decreased PCL) because of the inhomogeneous morphology.

The $T_{1} \mathrm{C}$ value can provide information on molecular motions for each substituent because its value is less affected by ${ }^{1} \mathrm{H}$ spin diffusion than the $T_{1} \mathrm{H}$ value. Actually, the $T_{1} \mathrm{C}$ change for each substituent as a function of the PCL content showed a different trend from the $T_{1} \mathrm{H}$ change (Fig. $6 \mathrm{~B}$ ). The $T_{1} \mathrm{C}$ values of the PHA moiety decreased with increasing PCL content: they attained minimum $T_{1} \mathrm{C}$ values at $70 \%$ PCL addition (4). In contrast, the $\alpha-\mathrm{CH}_{2}$ of PCL moiety obviously had a maximum $T_{1} \mathrm{C}$ value at $50 \%$ PCL addition (3). As did the $T_{1} \mathrm{C}$ values of preplasticized PHA $\left(\mathrm{CH}_{2}\right),{ }^{24}$ the $T_{1} \mathrm{C}$ values of PCL homopolymer (5) decreased with increasing temperature (Fig. S3, ESI $\dagger$ ). The $T_{1} \mathrm{C}$ value provides information about rapid molecular motions ( $\mathrm{MHz}$ order) of polymer constituents in plasticized PHA/PCL blends. Since the $T_{1} \mathrm{C}$ values of all substituents except $\mathrm{CH}_{3}$ group decreased with increasing temperature, the molecular motions connected with these $T_{1} \mathrm{C}$ values are associated with the long correlation time region $\left(\tau_{\mathrm{c}} \omega_{0} \gg 1\right) .{ }^{29}$ Therefore, for the components in plasticized PHA/PCL blends, the increase of $T_{1} \mathrm{C}$ equates to a decrease of molecular mobility of a $\mathrm{MHz}$ order, which matches with the Larmor frequency of ${ }^{13} \mathrm{C}$ nuclei. The $T_{1} \mathrm{C}$ changes associated with blending with $50 \%$ PCL show that the sea-island morphology in plasticized PHA/PCL blends considerably decreased the molecular mobility of PCL. In summary, the ${ }^{13} \mathrm{C}$ MAS NMR changes due to blending with PCL revealed that the $T_{1} \mathrm{C}$ decrease of PHA in plasticized PHA/PCL blends was caused by the increase of the molecular mobility due to the interaction between PHA and PCL.

Next, changes of $T_{1} \mathrm{H}$ values with the compatibilizers were examined for each polymer component in the 50\% plasticized PHA/50\% PCL blend (Fig. 6C). Interestingly, the long $T_{1} \mathrm{H}$ values 
A
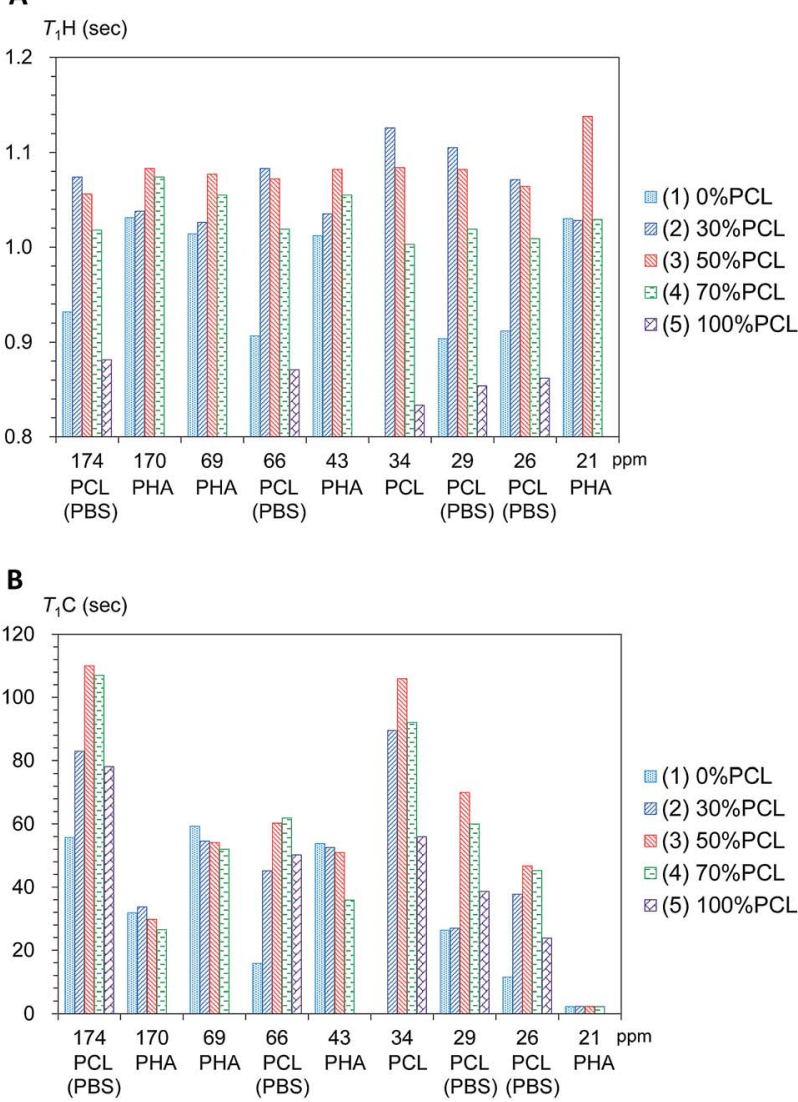

C

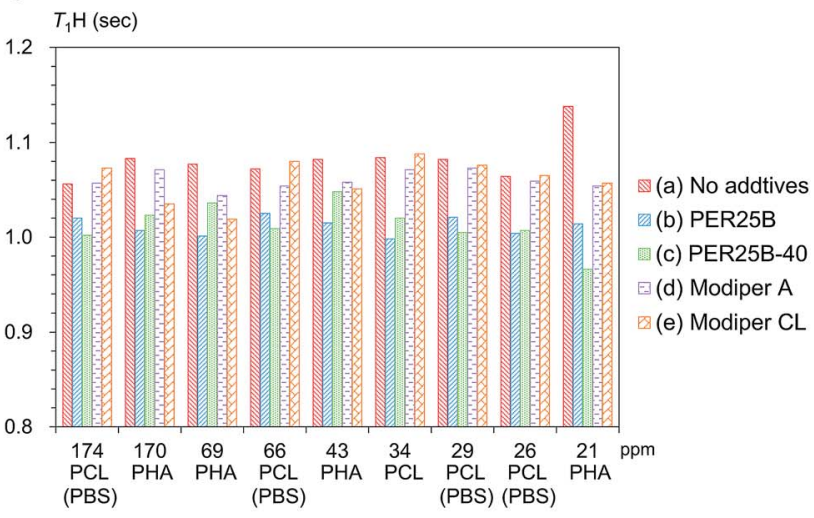

D

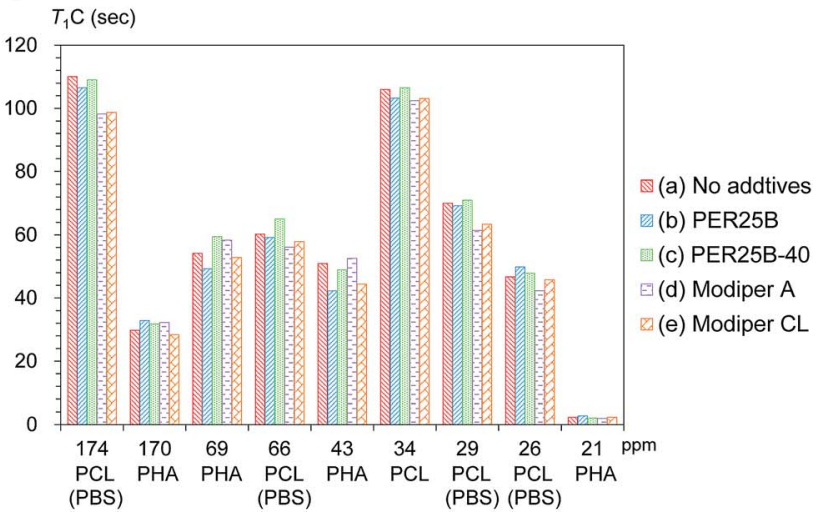

Fig. 6 Changes of $T_{1} H$ and $T_{1} C$ values of plasticized PHA/PCL blends. (A) $T_{1} H$ changes with PCL ratio. (B) $T_{1} C$ changes with PCL ratio. (C) $T_{1} H$ changes with compatibilizers. (D) $T_{1} \mathrm{C}$ changes with compatibilizers.

produced by the blending with PCL were shortened again by the addition of crosslinkers (PERHEXA 25B, PERHEXA 25B-40). The $T_{1} \mathrm{H}$ values of the $50 \%$ plasticized $\mathrm{PHA} / 50 \%$ PCL blend containing the crosslinkers had similar $T_{1} \mathrm{H}$ values to those of the plasticized PHA without PCL. Meanwhile, the graft polymers (Modiper A4400, Modiper CL430-G) produced almost unchanged $T_{1} \mathrm{H}$ values of the PCL and PBS moieties but slightly decreased $T_{1} \mathrm{H}$ values of the PHA moiety. The morphology observed by micro-focus X-ray CT with addition of compatibilizers is closely related to the change of the $T_{1} \mathrm{H}$ value. The crosslinkers produced a homogenous morphology that enhanced the $T_{1} \mathrm{H}$ relaxation of the polymers constituents while the graft polymers maintained the sea-island structure, resulting only in a small change in the $T_{1} \mathrm{H}$ relaxation of PHA.

The effect of compatibilizer on the $T_{1} \mathrm{C}$ values showed marked changes from the $T_{1} \mathrm{H}$ values, as shown in Fig. 6D. The crosslinkers (PERHEXA 25B, PERHEXA 25B-40) scarcely changed the $T_{1} \mathrm{C}$ values of the $50 \%$ plasticized PHA/50\% PCL blend, resulting in only small decreases for PHA $\left(\mathrm{CH}\right.$ and $\mathrm{CH}_{2}$ groups) using PERHEXA 25B. The graft polymers (Modiper A4400, Modiper CL430-G) caused $T_{1} \mathrm{C}$ decreases of the overlapped PCL/PBS signals in the $50 \%$ plasticized PHA/50\% PCL blend. Although both crosslinker and graft polymer changed mechanical properties, the mechanism by which these changes were manifested differed between crosslinker and graft polymer. That is, the crosslinkers changed morphology uniformly while producing little change in the molecular mobility of PHA, while the graft polymers increased the molecular mobility of PBS in the blend without changing the morphology.

\section{Spin-lattice relaxation in the rotational frame of plasticized PHA/PCL blends}

Due to the cross-polarization produced by the CP-MAS pulse sequence, the ${ }^{13} \mathrm{C}$ signal increased with the magnetization transfer from ${ }^{1} \mathrm{H}$ nuclei to ${ }^{13} \mathrm{C}$ nuclei and, at the same time, the ${ }^{13} \mathrm{C}$ signal decreased with the ${ }^{1} \mathrm{H}$ spin-lattice relaxation in the rotational frame $\left(T_{1 \rho} \mathrm{H}\right.$ relaxation). Therefore, the changes of $T_{1 \rho} \mathrm{H}$ relaxation can be monitored by the observation of the arrayed ${ }^{13} \mathrm{C}$ CP-MAS NMR spectra at various contact times.

Fig. 7 shows the $\mathrm{CH}_{2}$ signal intensity curves versus contact time for the plasticized PHA/PCL blends for various PCL contents. For the $\mathrm{CH}_{2}$ group, the $T_{1 \rho} \mathrm{H}$ relaxation of the PHA moiety was more enhanced with the addition of PCL, with the result that the ${ }^{1} \mathrm{H}$ spin-lattice relaxation time in the rotational frame $\left(T_{1 \rho} \mathrm{H}\right)$ of $\mathrm{PHA}-\mathrm{CH}_{2}$ decreased with increasing PCL content (Fig. 7A). Although the $T_{1 \rho} \mathrm{H}$ relaxation of PCL- $\alpha-\mathrm{CH}_{2}$ was suppressed in the $50 \% \mathrm{PHA} / 50 \%$ PCL blend, it proceeded more rapidly with increasing PCL content over 50\% (Fig. 7B). Furthermore, the $T_{1 \rho} \mathrm{H}$ relaxation of the overlapping $\mathrm{CH}_{2}$ 


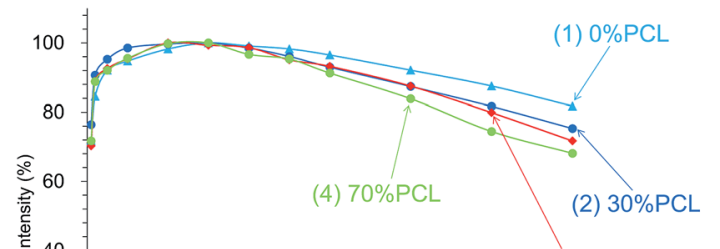

(3) $50 \% \mathrm{PCL}$
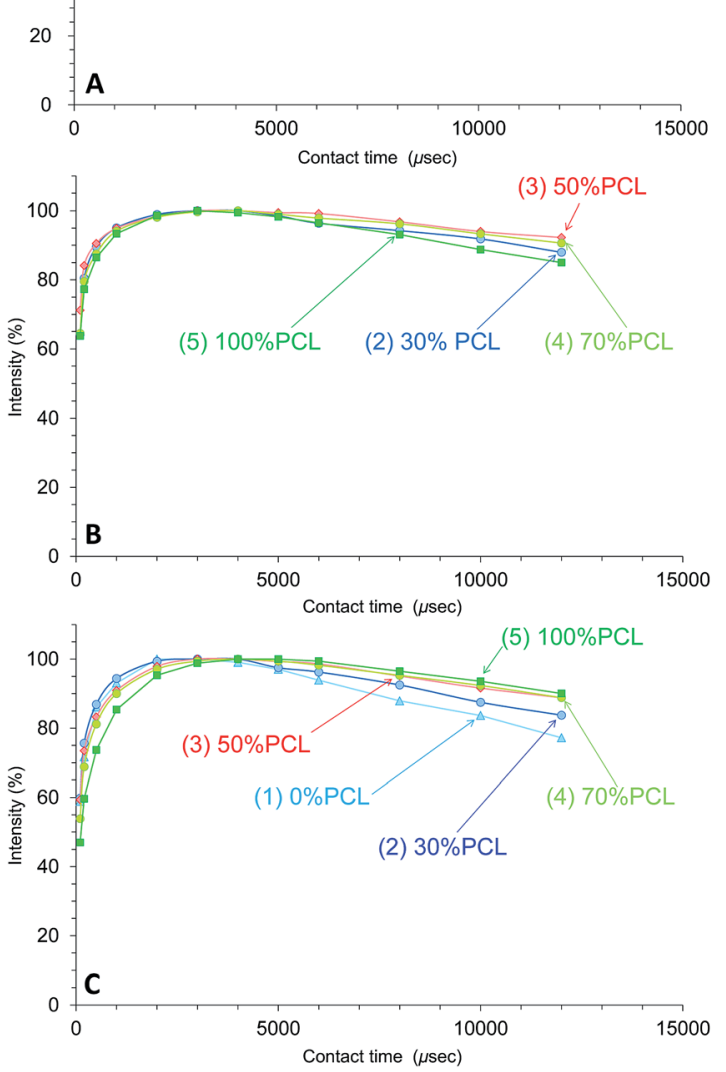

Fig. 7 Peak intensity changes of plasticized PHA/PCL blends versus contact time in ${ }^{13} \mathrm{C} C \mathrm{C}-\mathrm{MAS}$ NMR. (A) $\mathrm{PHA}, \mathrm{CH}_{2}$ (43 ppm). (B) $\mathrm{PCL}, \mathrm{CH}_{2}$ (34 ppm). (C) PBS-PCL, $\mathrm{CH}_{2}$ (25 ppm).

features of PBS/PCL was suppressed to various extents depending on the PCL content; that is, the $T_{1 \rho} \mathrm{H}$ of PBS decreased with increasing PCL content (decreasing PHA with PHA content) (Fig. 7C). Therefore, the addition of PCL into the plasticized PHA (the PHA/PBS copolymer) suppressed the $T_{1 \rho} \mathrm{H}$ relaxation of PBS (long $T_{1 \rho} \mathrm{H}$ value), which compensated for the enhanced $T_{1 \rho} \mathrm{H}$ relaxation of PHA (short $T_{1 \rho} \mathrm{H}$ value) resulting from the immiscible morphology as well as the $T_{1} \mathrm{H}$ relaxation.

In contrast to the effect of PCL addition, the $T_{1 \rho} \mathrm{H}$ relaxation changed little with various compatibilizers, as shown in Fig. 8. The $T_{1 \rho} \mathrm{H}$ relaxation of $\mathrm{PHA}-\mathrm{CH}_{2}$ was slightly suppressed by the compatibilizers, especially by Modiper CL (Fig. 8A). For $\mathrm{CH}_{2}$ groups in both PCL (Fig. 8B) and overlapping signals from PBS/ PCL (Fig. 8C), neither crosslinker or graft polymer produced any significant change in the signal decay curve. That is, the compatibilizers slightly lengthened the $T_{1 \rho} \mathrm{H}$ value of PHA while they scarcely changed the $T_{1 \rho} \mathrm{H}$ values of PCL and PBS. We conclude that $T_{1 \rho} \mathrm{H}$ relaxation is concerned with slow molecular mobility (kHz order) and is not related to the tensile properties

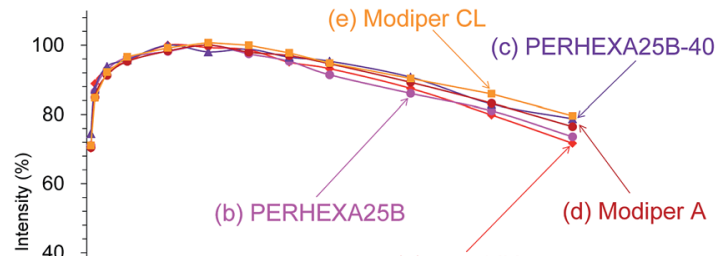

(a) no additives
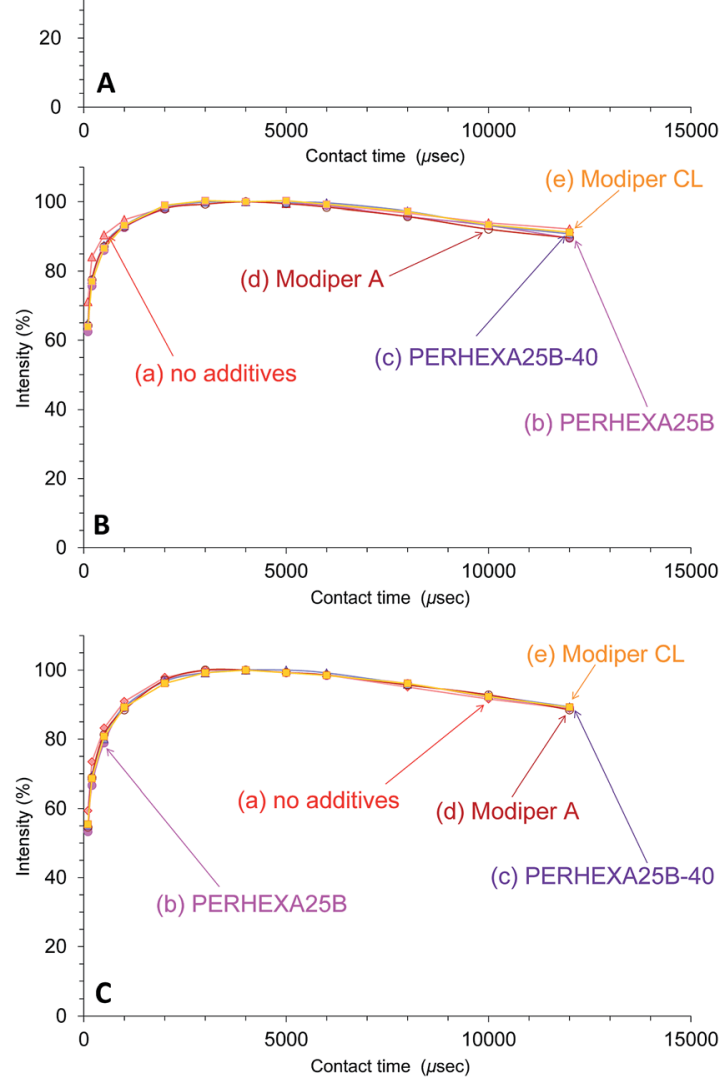

Fig. 8 Peak intensity changes of $50 \%$ plasticized $\mathrm{PHA} / 50 \% \mathrm{PCL}$ blends versus contact time in ${ }^{13} \mathrm{C} C \mathrm{CP}-\mathrm{MAS}$ NMR. (A) $\mathrm{PHA}, \mathrm{CH}_{2}$ (43 ppm). (B) $\mathrm{PCL}, \mathrm{CH}_{2}$ (34 ppm). (C) PBS-PCL, $\mathrm{CH}_{2}(25 \mathrm{ppm})$.

but could be an indicator of interactions within constituent polymers of the PHA-based blend.

\section{Multi-scale instrumental analyses of PHA/PCL blends for the effects of blend ratio and additives}

Based on the micro-focus X-ray CT observation (Fig. 2), Fig. 9 presents schematics of the nanostructural changes of the $50 \%$ plasticized PHA/50\% PCL blend with/without the compatibilizers after the tensile test. Without compatibilizers [Fig. 9(A)], the blend did not interact with PCL and formed the sea-island morphology seen before tensile tests (a). Although PBS in the blend had a similar chemical structure to PCL, the interaction between the plasticized PHA and PCL was weak in the sea-island morphology. In the quasi-static test (b), the plasticized PHA was difficult to extend, even though PCL readily extended. Because of the weak interaction between the plasticized PHA and PCL, the plasticized PHA in the blend adopted a broken island morphology and exfoliated to form the interface with PCL. In 
A

(a)

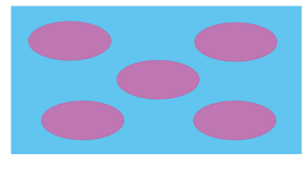

B

(a)

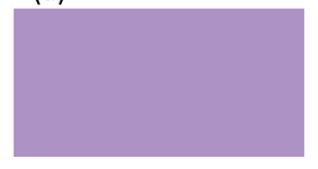

C

(a)

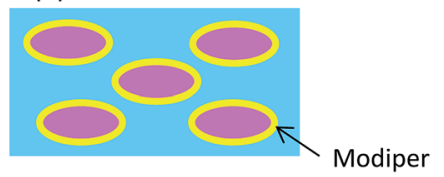

(b)

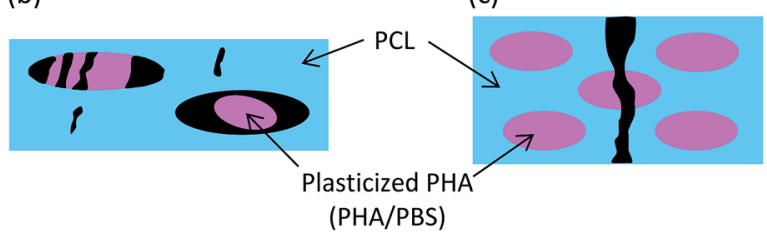

(b)

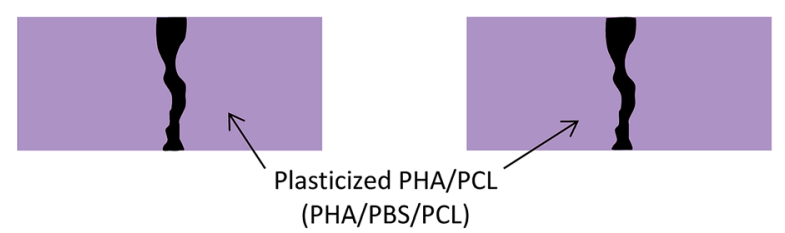

(b)

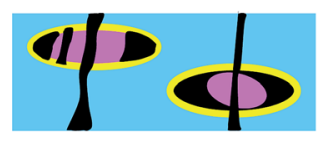

(c)

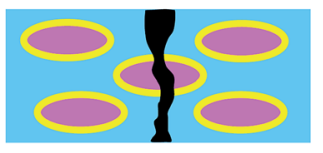

Fig. 9 Nanostructural changes of 50\% plasticized PHA/50\% PCL blend after tensile tests. (A) No additives, (a) before tensile test, (b) after quasistatic test, (c) after dynamic test. (B) Crosslinker, (a) before tensile test, (b) after quasi-static test, (c) after dynamic test. (C) Graft copolymer, (a) before tensile test, (b) after quasi-static test, (c) after dynamic test.

the dynamic tensile test (c), since PCL was also difficult to extend, the $50 \%$ plasticized PHA $/ 50 \%$ PCL blend was easily broken at a small elongation.

By the addition of a crosslinker into the $50 \%$ plasticized PHA/50\% PCL blend [Fig. 9(B)], the plasticized PHA became compatible with PCL (a). Both plasticized PHA and PLC integrated to produce a homogenous morphology over tens of micron. In the quasi-static test (b), the unification of the plasticized PHA and PCL restrained stretching of the PCL. The plasticized PHA/PLC blend was easily broken at a small elongation because the quasi-static test was dominated by the rigidity of the PHA moiety. Furthermore, the elongation at break in the dynamic tensile test (c) was improved by the addition of cross-linker because the dynamic test was dominated by the flexibility of the PCL moiety.

On the addition of the graft polymer [Fig. 9(C)], although the $50 \%$ plasticized PHA $/ 50 \%$ PCL blend still adopted the seaisland morphology, the interaction between the plasticized PHA and PCL became strengthened (a), as confirmed by the relaxation time analyses. However, the unifying effect of the graft polymer on the plasticized PHA/PLC blend was weaker than with the crosslinker. Therefore, both plasticized PHA and PCL were broken with a larger elongation at break in the quasistatic test (b). Since the unification of the plasticized PHA/PLC blend mobilized the plasticized PHA and PCL together, the elongation at break was also increased by the addition of graft polymer in the dynamic test (c).

\section{Conclusions}

With the aim of extending application of multi-scale instrumental analyses to biodegradable polymers, plasticized polyhydroxyalkanoates (PHA), containing $65 \%$ PHA, 30\% pol$y$ (butylene succinate) (PBS), and $5 \%$ crosslinker, were examined we respect to blending with poly ( $\varepsilon$-caprolactone) (PCL) as well as the addition of compatibilizers, such as crosslinkers and graft polymers. Blending PHA with more than 50\% PCL increased the tensile strength and elongation at break in the quasi-static tensile test, although the tensile properties were independent of PCL content in the impact tensile test. The crosslinkers markedly lowered the elongation at break in the quasi-static tensile test, while the graft polymers only produced a slight decrease. Both crosslinkers and graft polymers increased the tensile strength and elongation at break in the impact tensile test. Micro-focus X-ray CT observations revealed a sea-island morphology in the $50 \%$ plasticized PHA $/ 50 \%$ PCL blend. Although the morphology was slightly changed by the addition of graft polymers having acrylonitrile-styrene copolymers as a side-chain, it became homogenous over distances of more than ten microns with the addition of peroxy crosslinkers. The combination of ${ }^{13} \mathrm{C} \mathrm{CP}$ - and PST-MAS NMR spectra showed that the molecular mobility of PCL was suppressed by blending with the plasticized PHA while the compatibilizers left the molecular motions almost unchanged, with the exception of the $\mathrm{C}=\mathrm{O}$ groups of PBS/PCL. The ${ }^{1} \mathrm{H}$ spin-lattice relaxation time $\left(T_{1} \mathrm{H}\right)$ values of the plasticized PHA/PCL blend increased in the seaisland morphology at 50\% PCL addition. The lengthened $T_{1} \mathrm{H}$ value of the $50 \%$ plasticized PHA $/ 50 \%$ PCL blend was shortened again by the crosslinkers, which also changed the sea-island morphology into a homogenous mixture. The $T_{1} \mathrm{H}$ value of blend was shortened to a smaller degree by the graft polymers, which also produced less change in the sea-island morphology. Blending with the plasticized PHA increased the ${ }^{13} \mathrm{C}$ spin-lattice relaxation time $\left(T_{1} \mathrm{C}\right)$ value of PCL, indicating that the sea-island 
morphology decreased the molecular mobility of the added PCL. Meanwhile, the $T_{1} \mathrm{C}$ values of the PHA moiety decreased with increasing PCL content to indicate that the added PCL amplified the molecular mobility of PHA. Concerning the effects of compatibilizer on the $T_{1} \mathrm{C}$ values, the graft polymers noticeably decreased the $T_{1} \mathrm{C}$ value of the PBS constituent. The ${ }^{1} \mathrm{H}$ spin-lattice relaxation in the rotational frame $\left(T_{1 \rho} \mathrm{H}\right.$ relaxation) of PHA was enhanced by suppression of the $T_{1 \rho} \mathrm{H}$ relaxation of PBS because of the immiscible morphology, while the $T_{1 \rho} \mathrm{H}$ relaxation was little changed by the addition of compatibilizers. The molecular mobility of the polymers constituents in the plasticized PHA was changed by blending with PCL and the change of molecular mobility caused the increase of tensile strength and elongation at break in the quasi-static tensile test. In contrast, the molecular mobility of the blend was not changed by the crosslinker but was by the graft polymer, while the morphology was not changed by the graft polymer but was by the crosslinker. For these reasons, a different trend for the tensile properties in quasi-static and impact tests was observed when using either crosslinkers or graft polymers. In conclusion, to improve the tensile properties of biodegradable polymers, it is necessary not only to add a component having superior tensile properties but also to match the interaction and molecular mobility of the new component with the original polymer. Considering the tensile properties from the interactions between the constituent polymers, the graft polymer was suitable for polymer blends having different molecular mobility, such as plasticized PHA/PCL blends. In contrast, the crosslinker would be more suitable for polymer blends having similar molecular mobility to each other. NMR is a good indicator of success as the $T_{1} \mathrm{H}$ and $T_{1 \rho} \mathrm{H}$ values, which are the indexes involved in the tensile properties, decrease with an increasing interaction while the $T_{1} \mathrm{C}$ value decrease with increasing the molecular mobility. Our present study showed that the multi-scale instrumental analyses gave information about molecular mobility and morphology concerned with the tensile properties of synthetic polymers. Studies of materials characteristics are currently in progress for not only synthetic polymers, but also for natural polymers, using multi-scale instrumental analyses.

\section{Conflicts of interest}

There are no conflicts to declare.

\section{Acknowledgements}

The authors wish to express special thanks to Kohei Yasuda, Nagoya Institute of Technology, for help with the tensile test data analyses.

\section{References}

1 S. Chanprateep, J. Biosci. Bioeng., 2010, 111, 621.

2 X. Gao, J.-C. Chen, Q. Wu and G.-Q. Chen, Curr. Opin. Biotechnol., 2011, 22, 768.
3 Z. A. Raza, S. Abid and I. M. Banat, Int. Biodeterior. Biodegrad., 2018, 126, 45.

4 E. Bugnicourt, P. Cinelli, A. Lazzeri and V. Alvarez, eXPRESS Polym. Lett., 2014, 8, 791.

5 B. Laycock, P. Halley, S. Pratt, A. Werker and P. Lant, Prog. Polym. Sci., 2013, 38, 536.

6 Z. A. Raza, S. Riaz and I. M. Banat, Biotechnol. Prog., 2018, 34, 29.

7 D. Kai and X. J. Loh, ACS Sustainable Chem. Eng., 2014, 2, 106.

8 H. Verhoogt, B. A. Ramsay and B. D. Favis, Polymer, 1994, 35, 5155.

9 I. Zembouai, M. Kaci, S. Bruzaud, A. Benhamida, Y.-M. Corre and Y. Grohens, Polym. Test., 2013, 32, 842.

10 I. Armentano, E. Fortunati, N. Burgos, F. Dominici, F. Luzi, S. Fiori, A. Jiménez, K. Yoon, J. Ahn, S. Kang and J. M. Kenny, LWT-Food Sci. Technol., 2015, 64, 980.

11 M. P. Arrieta, J. López, D. López, J. M. Kenny and L. Peponi, Eur. Polym. J., 2015, 73, 433.

12 M. Larsson, O. Markbo and P. Jannasch, RSC Adv., 2016, 6, 44354.

13 D. Garcia-Garcia, J. M. Ferri, T. Boronat, J. Lopez-Martinez and R. Balart, Polym. Bull., 2016, 73, 3333.

14 Y. Ke, X. Y. Zhang, S. Ramakrishna, L. M. He and G. Wu, Mater. Sci. Eng., C, 2017, 70, 1107.

15 Y.-X. Zhou, Z.-G. Huang, X.-Q. Diao, Y.-X. Weng and Y.-Z. Wang, Polym. Test., 2015, 42, 17.

16 P. Ma, D. G. Hristova-Bogaerds, P. J. Lemstra, Y. Zhang and S. Wang, Macromol. Mater. Eng., 2012, 297, 402.

17 A. Chikh, A. Benhamida, M. Kaci, I. Pillin and S. Bruzaud, Polym. Test., 2016, 53, 19.

18 Y. Parulekar and A. K. Mohanty, Macromol. Mater. Eng., 2007, 292, 1218.

19 F. G. Morin and R. H. Marchessault, Macromolecules, 1992, 25, 576.

20 A. Cao, N. Asakawa, N. Yoshie and Y. Inoue, Polymer, 1999, 40, 3309.

21 L. Zhang, H. Tang, G. Hou, Y. Shen and F. Deng, Polymer, 2007, 48, 2928.

22 M. Nishida, T. Tanaka, T. Miki, Y. Hayakawa and K. Kanayama, Cellulose, 2017, 24, 5295.

23 M. Nishida, T. Tanaka, T. Miki, T. Ito and K. Kanayama, Ind. Crops Prod., 2018, 114, 1.

24 M. Nishida, T. Tanaka, Y. Hayakawa and M. Nishida, Polymers, 2018, 10, 506.

25 M. Nishida, T. Ogura, H. Shinzawa, M. Nishida and W. Kanematsu, J. Mol. Struct., 2016, 1124, 92.

26 M. Nishida, Y. Ito, H. Shinzawa, M. Nishida and Y. Hayakawa, Key Eng. Mater., 2016, 715, 39.

27 M. Nishida, H. Ichihara, H. Watanabe, N. Fukuda and H. Ito, in Dynamic Behavior of Materials, ed. V. Chalivendra, B. Song and D. Casem, Springer New York, 2012, vol. 1, p. 329.

28 D. A. Torchia, J. Magn. Reson., 1978, 30, 613.

29 M. Nishida, T. Tanaka, T. Miki, Y. Hayakawa and K. Kanayama, RSC Adv., 2017, 7, 54532. 